\title{
Finite Element Analysis of the Meniscectomised Tibio-Femoral Joint: Implementation of Advanced Articular Cartilage Models
}

\author{
Lorenza Mattei ${ }^{1,2^{*}}$, Eleonora Campioni ${ }^{1,3}$, Mario Alberto Accardi ${ }^{1}$ and Daniele Dini ${ }^{1}$ \\ ${ }^{1}$ Department of Mechanical Engineering, Imperial College London, South Kensington Campus, Exhibition Road, \\ London SW7 2AZ, UK \\ 2Department of Mechanical, Nuclear and Production Engineering, University of Pisa, Largo Lucio Lazzarino 1, Pisa, \\ 56100 , Italy \\ ${ }^{3}$ Department of Mechanical and Civil Engineering, University of Modena and Reggio Emilia, Via Vignolese, 905/b, \\ 41125 Modena, Italy
}

\section{ABSTRACT}

The article presents advanced computer simulations aimed at the accurate modelling of human tibio-femoral joints in terms of anatomy, physiological loading, and constitutive behaviour of the tissues. The main objective of this research is to demonstrate the implications that the implementation of different articular cartilage models have on the prediction of the joint response. Several biphasic material constitutive laws are tested using a finite element package and compared to the monophasic linear elastic description, often still used to predict the instantaneous response of the cartilage in threedimensional knee models. Thus, the importance of adequately capturing the contribution of the interstitial fluid support is proved using a simplified three-dimensional model; subsequently, a biphasic poroviscoelastic non linear constitutive law is implemented to study the response of a patient specific tibio-femoral joint subjected to simplified walking cycles. The time evolution of stresses, pore pressure, contact areas and joint displacements are captured and compared with existing meniscectomised knee models. Contact pressures and areas obtained using the developed numerical simulations are in agreement with the existing experimental evidence for meniscectomized human knee joints. The results are then used to predict the most likely site for the origin of mechanical damage, i.e. the medial cartilage surface for the specific case analysed in the present contribution. Finally, future research directions are suggested.

Keywords: Articular Cartilage; Biphasic; Fluid load support; Tibio-femoral joint; Finite Element Modelling; Osteoarthritis.

\section{Introduction}

Osteoarthritis $(\mathrm{OA})$ is a degenerative joint condition which causes irreversible damage to the structural and the mechanical integrity of articular cartilage (AC). Osteoarthritic AC differs from healthy $A C$ in that mechanically, it tends to gradually become softer, and visually, fibrillation and shallow fissures on the surface start to occur (Buckwalter and Mankin 1998, 2006). Eventually, at later stages of the pathology, ulceration and full AC loss occurs. Consequently osteoarthritic AC loses its fundamental capability to protect bone extremities and to attenuate loads, causing pain and limiting patient's life quality.

This pathology is today of great interest since it is thought to affect $85 \%$ of people by the age of 55 (Fergusson 1987, Wilson et al. 2005b) and recognized as the primary cause of joint replacement surgery. At present a well accepted classification distinguishes between primary and secondary OA. Primary OA is referred to as OA which develops in apparently normal joints with absence of any joint degeneration (e.g. healthy ligaments and menisci) and with no predisposing factors (Buckwalter and Martin 2006), and prevails in older adults. Secondary OA can be triggered by abnormal and excessive stresses in the tissue (Wilson et al. 2005b) caused by trauma, injury, hereditary or inflammatory factors (Buckwalter and Martin 2006). The latter is the subject of our investigation in the present contribution. In particular, meniscectomy is well recognized as risk factor for secondary OA in the knee (Papalia et al. 2011) causing a severe change in the knee contact mechanics. As for example, the loss of the medial meniscus leads to a decrease of up to $75 \%$ and an increase of up to $300 \%$ in contact area and contact stress, respectively (Baratz et al. 1986, Ihn et al. 1993, Kurosawa et al. 1980, McDermott and Amis 2006). Many clinical studies have highlighted the significant incidence of OA after meniscectomy, which can be observed both in the short and in the long term follow-up (Englund and Lohmander 2004, Petty and Lubowitz 2010, Roos et al. 2001), and affects both young (McNicholas et al. 2000) and older patients (Crevoisier et al. 2001). In addition to this, it has been demonstrated that total meniscectomy causes higher incidence of degenerative change compared to partial meniscectomy (Hede et al. 1992, McGinty et al. 1997). In-vitro studies on animals have confirmed this evidence: meniscectomy-induced OA models revealed

\footnotetext{
*Corresponding Author, Email: I.mattei@ing.unipi.it
} 
biological, bio-chemical, and biomechanical degradation of knee articular cartilage centrally, and osteophyte formation peripherally (Appleyard et al. 2003, LeRoux et al. 2000, Oakley et al. 2004, Song et al. 2008, Young et al. 2006).

Secondary OA, being mechanically induced, has recently become the subject of many biomechanical and biomedical engineering studies. To this aim finite element method (FEM) is certainly an invaluable tool for studying stresses and strains distribution within healthy or injured knee joints, and investigating the onset of $A C$ damage. However, this can only be successful if suitable and realistic constitutive models of $A C$ are employed. In the last decades a plethora of advanced constitutive models for AC have been proposed and numerically implemented using FEM in order to describe the unique mechanical behaviour of AC dictated by its biphasic nature (Mow et al. 1980), flow-dependent and independent viscoelasticity (DiSilvestro, Zhu and Suh 2001, DiSilvestro, Zhu, Wong et al. 2001), and its strong structural and mechanical anisotropy mainly due to fibril reinforcement (Hosoda et al. 2010, Julkunen et al. 2008, Li et al. 2009, Wilson et al. 2005a).

Although a linear elastic model is a long way away from an accurate representation of $A C$, it has been largely adopted in the study of knee biomechanics. Indeed, the high non linearity of both realistic knee geometries and AC mechanical behaviour lead to define a numerical problem of very complex nature and computationally expensive. Consequently, the linear elastic model was adopted to model AC in several patient specific finite element (FE) models of the knee used for investigating stresses and strains of native (Beillas et al. 2004, Bendjaballah et al. 1997, Donahue et al. 2002, Guo et al. 2009, Moglo and Shirazi-Adl 2005, Papaioannou et al. 2008, Pena et al. 2006) and meniscectomised (Bendjaballah et al. 1995, Pena et al. 2008, Pena et al. 2005, Shirazi and Shirazi-Adl 2009, Yang et al. 2010) joints. Only very recently, sophisticated biphasic constitutive models of AC have been implemented in human knee FE models. The fibril-reinforced model proposed by Li et al. (2009) has been implemented in knee FE models ${ }^{\dagger}$ (Gu and Li 2011, Kazemi et al. 2011) in order to investigate the effect of pore pressure and fiber orientation in contact mechanics of both healthy and meniscectomised knee joints (Kazemi et al. 2011). The effect of the fibril pattern has also been investigated by Mononem and co-workers both for healthy and osteoarthritic AC in human knee (Mononen et al. 2012). In this case the fibril-reinforced poroviscoelastic model described in (Julkunen et al. 2008, Mononen et al. 2011, Wilson et al. 2005a) was adopted for AC. The models mentioned above simulated compression loads with the femur in full-extension, i.e. a fast loading ramp followed by a constant load. The maximum load was relatively low in (Gu and Li 2011) and (Kazemi et al. 2011), being $36 \mathrm{~N}$ and $300 \mathrm{~N}$ (in 1 s) respectively, whilst a physiological load of $1000 \mathrm{~N}$ (in $1 \mathrm{~s}$ ) was applied in (Mononen et al. 2012). In addition, the majority of these models rely on the implementation of laws for the description of the tissue's constitutive behaviour by the means of user defined material subroutines, which are often not readily available or simple to code and usually are linked to high computational costs.

The aim of the research presented in this article is threefold: (i) to develop a patient-specific meniscectomised knee joint model to be used to assess the contact stresses induced by realistic loading histories; (ii) to perform a critical comparison between linear elastic and readily available biphasic constitutive models applied to the study of joints biomechanics; and (iii) to estimate the likelihood of articular cartilage (AC) damage to occur and the location of damage initiation.

To serve this purpose, a realistic FE model of the tibio-femoral joint (TFJ) contact following total meniscectomy has been reconstructed from MRI and CT scans of a human knee joint. This model is used to provide further evidence that linear elastic constitutive laws should not be used to model AC if the instantaneous response of the tissue is under investigation. Indeed this topic is still object of an active debate in the scientific community, as confirmed by recent publications (see e.g. ( $\mathrm{Li}$ and $\mathrm{Gu}$ 2011)). On the other hand, biphasic constitutive descriptions should be used, and here a number of biphasic laws are tested using a commercial package to establish which one provides the best results in comparison with the existing experimental evidence collected on cartilage samples tested under physiological conditions. It should be noted that, although the incorporation of fibril-reinforced non linear models have already been successfully attempted for 2D configurations by our group (Accardi and Dini 2009), the non linearity in the elastic behaviour of the solid constituents of the AC tissue is not explicitly modelled in the research presented here. This is due to the fact that the incorporation of the non linear response due to the AC fibrils network requires user-defined material description to be implemented in the adopted finite element package (see e.g. (Gu and Li 2011, Kazemi et al. 2011, Mononen et al. 2012)); this in turn implies that (a) the model is not as readily portable as those developed using the constitutive laws tested in this study (which do not require user-defined materials for their implementation), (b) the computational time increases significantly, especially if also the viscoelastic contribution of the fibrils network is considered, (c) simulating repeated loading cycles under physiological conditions is difficult, even using reasonably large computational resources. Therefore, also bearing in mind that one of our aims was to simulate realistic loading scenarios, simpler biphasic models, which still capture the fluid-induced non linearity and the viscoelasticity of the solid matrix, and provide stress maps for repeated loading cycles in a reasonable amount of CPU time have been favoured in this study.

The paper is outlined as follows. First, in Section 2, we introduce and thoroughly discuss different AC constitutive models. These models include: (i) the biphasic isotropic model with linear permeability (BL), (ii) the biphasic isotropic

\footnotetext{
† It should be noted that viscoelastic effects have generally been neglected in these studies.
} 
model with non linear permeability (BNL), (iii) the transversely isotropic biphasic model (BTINL) and (iv) the poroviscoelastic model (BPVNL). The effect of the implementation of different AC models in a simplified threedimensional configuration is then explored in Section 3. In Section 4, a patient specific FE model of the TFJ is developed in order to compare the effect of monophasic and biphasic models of AC on the joint mechanical response and to investigate the effect of physiological loading-unloading cycles when reproducing simplified walking conditions. Finally, the results obtained from the models described in Sections 3 and 4 are discussed in Section 5, and conclusions are drawn in Section 6.

\section{Constitutive models for AC}

This section describes the constitutive laws and the methods employed by the authors in the present study to investigate the mechanical response of $\mathrm{AC}$; this is done in order to explore the suitability of the existing constitutive material behaviours to the study of realistic TFJ geometries.

The anatomical structures to be considered and modelled when analysing the human joints under investigation consist of menisci, ligament, muscles, cortical bone, trabecular bone and the AC. In this study, the presence of the ligaments and the muscles were reproduced implicitly using ad-hoc boundary conditions (BCs), while the presence of the menisci was ignored as our focus is placed on the meniscectomised knee joints. As far as the mechanical properties of the materials modelled are concerned, the femur and the tibia extremities are assumed to be composed of only epiphyseal surface bone, which has mechanical properties in the range between the cortical and trabecular bone's properties. These are therefore modelled as homogeneous, isotropic and linear elastic, with a Young's modulus of 5 GPa and a Poisson's ratio of 0.3 (Beillas et al. 2004).

The real focus of this study is the AC tissue, a very complex material with a unique mechanical behaviour mainly due to its biphasic nature. In its most common representation, it consists of two distinct phases (Mow et al. 1980): a porous solid matrix phase and an interstitial fluid phase. The solid matrix, often referred to as extra cellular matrix $(E C M)$, which is mainly composed of a collagen fibril network, proteoglycans (PGs), and glycoproteins (Schneck and Bronzino 2002). The interstitial fluid phase contains approximately $75 \%$ water (Rieppo et al. 2004). The AC components, including the proportion of interstitial fluid, are distributed in different quantities and with different architectures within the AC thickness, making the tissue strongly inhomogeneous and anisotropic. In addition, AC exhibits a significant viscoelastic response, which is in turn characterised by both flow dependent and flow independent components. The flow dependent viscoelasticity is caused by the frictional drag force caused by the fluid flow through the ECM which is controlled by the permeability. Indeed, in porous media, the permeability is such that it is not constant but decreases with compressive strain due to the reduction of the porous matrix void ratio (the ratio between the fluid and solid fractions). As a consequence, water can move in and out of the tissue, therefore varying, as a function of the applied load/strain history, the fraction of the load supported by the solid and the fluid constituents. The flow independent viscoelasticity corresponds to the intrinsic viscoelasticity of the solid matrix and it is mainly due to the PGs and the collagen fibrils. The latter show a strain dependent stiffness response, characterised by increasing stiffness with increasing tensile strain (Mak 1986). In AC tissue, a swelling behaviour is also observed, mainly caused by the negative charge of the PGs, which induces a chemical expansion phenomenon (due to the repulsion force between the tightly packed PGs) and the osmotic swelling (due to the higher ionic concentration of the ECM with respect to the surrounding synovial fluid) (Grodzinsky et al. 1981, Lai et al. 1991).

In the literature many material models of $A C$ can be found (Wilson et al. 2005b), ranging from the relatively simple biphasic models to models considering more or less explicitly all the main components of the tissue, such as the fibril reinforced models ( $\mathrm{Li}$ et al. 2009, Li et al. 1999, Soulhat et al. 1999, Wilson et al. 2005a) and triphasic models (Lai et al. 1991, Lu et al. 2010, Wu and Herzog 2002). In the present study only some of the existing biphasic AC models are considered and implemented in a commercial finite element software package. These models, while not explicitly modelling the microstructural features of AC, are chosen as they allow a realistic representation of the tissue's mechanical response, they are relatively simple to implement as they do not require the implementation of user defined material descriptions, and enable to run simulations of 3D non linear models at a relatively low computational cost.

The AC models, which have been considered in this study and implemented in the models described in Sections 3 and 4 , are briefly described below.

\section{$2.1 \quad$ Monophasic model}

The monophasic (M) model is the simplest model considered by researchers in this field (Bei and Fregly 2004, Beillas et al. 2004, Bendjaballah et al. 1997, Bendjaballah et al. 1995, Donahue et al. 2002, Donahue et al. 2003, Papaioannou et al. 2008, Pena et al. 2008, Pena et al. 2005): here, AC is modelled as a single solid phase, which is considered as linear elastic, homogenous, isotropic and almost incompressible. The model is thus characterised by only two mechanical parameters: the Young's modulus and the Poisson's ratio. In particular, instantaneous (i.e. 
dynamic) values of these two parameters are employed to represent the constitutive behaviour of the material; these are termed $E_{i n s}$ and vins, respectively. The instantaneous Young's modulus is extrapolated from indentation or compression curves obtained instantaneously after the load application on the AC and takes into account both the response of the solid and the fluid constituent. Consequently it accounts for the stiffness changes induced by the interstitial fluid pressurisation of $A C$ and increases with the strain rate. If enough time is allowed, however, equilibrium conditions are reached and $E_{\text {ins }}$ and $v_{\text {ins, }}$, correspond to the solid matrix parameters, $E_{\text {eq }}$ and $v_{e q}$.

\subsection{Biphasic linear model}

The biphasic linear (BL) model is the most basic of the biphasic models, initially developed by Torzilli and Mow (1976a, 1976b). It is the backbone of all the more advanced AC models. The tissue is assumed to be constituted by a linear elastic, homogenous, isotropic and almost incompressible solid phase and an incompressible fluid phase. The permeability is assumed to be constant (to which the linearity of the model is due), hence independent of the void ratio. All the biphasic models use the Young's modulus evaluated at equilibrium, $E_{\text {eq. }}$.

The BL model is acceptable as a general approximation of the AC mechanical behaviour since it enables to capture the main characteristics of the tissue due to its biphasic nature. Furthermore it is fairly simple to implement using FEM and not too computationally expensive. However, although capable of dealing with the long term creep and stress relaxation of AC during confined compression, this model shows non negligible deviations from the tissue's experimental response. The model becomes even more inaccurate when applied to unconfined compression tests, mainly due to the fact that the anisotropy of the tissue is neglected. As a consequence, the BL model tends to underestimate the stresses in the tissue, and this might constitute a severe limitation when investigating damage initiation (Armstrong et al. 1984, Brown and Singerman 1986).

\subsection{Biphasic non linear model}

The biphasic non linear (BNL) model combines the biphasic linear (BL) model with a non linear permeability law, thus reproducing the flow dependent viscoelasticity of the tissue. Lai et al. (1981) were the first to propose a non linear law to be used to account for the change in permeability related to the deformation of the porous matrix by specifying a relationship between the permeability and the void ratio. This law was later revisited by Van der Voet (1997), assuming the following form

$$
k=k_{0}\left(\frac{1+e}{1+e_{0}}\right)^{M}
$$

where $k_{0}$ and $M$ are material constants, and $e$ and $e_{0}$ the initial and the current void ratio, respectively.

It should be noted that the non linear permeability law proposed by Lai et al. (1981) provides a good description of the AC response only for small strains. Its adoption in the present study is justified by the fact that the AC tissue modelled by the authors undergo small deformations. However, a generalized form of this non linear permeability law has been proposed by Holmes and Mow (1990); since its validity has been demonstrated for both small and finite strains, such formulation should be adopted when larger loads are applied and larger strains are computed.

\subsection{Biphasic poroviscoelastic non linear model}

The biphasic poroviscoelastic non linear (BPVNL) model takes into account both the flow dependent and the flow independent contributions to the viscoelastic tissue response. The BPVNL model was first analytically formulated by Mak (1986) and implemented numerically using two different methods, namely the continuous (Fung 1972) and the discrete (Suh and Bai 1998) spectrum algorithms. The latter is more widely used for the viscoelastic approximation as it saves significant memory and CPU time, being equally reliable, and thus it is adopted in this study, where minimising the use of computational resources is imperative. The discrete spectrum algorithm describes viscoelastic properties using the reduced relaxation function $G(t)$ written as a series of combinations of discrete relaxations function $G_{i}$

$$
G(t)=G_{\infty}+\sum_{i=0}^{N_{d}} G_{i} e^{-t / \tau_{i}}
$$

where $\tau_{i}$ is the discrete relaxation time constant and $G_{\infty}=G(\infty)=1$ (Suh and Bai 1998). According to (Suh and Bai 1998) in the implementation of Eq. (2), it can be assumed $G_{i}=\breve{G}$, where $\breve{G}$ is the discrete spectrum magnitude and is 


$$
\breve{G}=\frac{G_{0}-1}{N_{d}+1}
$$

where $G_{0}=G(0)$. Furthermore $\tau_{i}$ can be considered equally distributed with a logarithmic interval between the short term and long term relaxation time constants, i.e. $\tau_{S}$ and $\tau_{L}$, and thus

$$
N_{d}=\log \left(\frac{\tau_{L}}{\tau_{S}}\right)
$$

It is worth noting that three additional parameters, i.e. $\breve{G}, \tau_{S}$ and $\tau_{L}$, are necessary to characterize the biphasic poroviscoelastic materials with respect to the biphasic model.

The BPVNL model is capable of representing the tissue's response and reproduce the experimental data obtained using confined and unconfined compression, and indentation tests. In particular, it can predict simultaneously lateral displacements and reaction forces during unconfined compression tests (DiSilvestro, Zhu, Wong et al. 2001) (DiSilvestro, Zhu, Wong et al. 2001), also for different strain rates (DiSilvestro, Zhu and Suh 2001). Although this model does include the fundamental characteristic of the flow-independent viscoelasticity, it does not capture anisotropy and the compression-tension non linearity of the tissue.

\subsection{Biphasic transversely isotropic linear model}

As previously discussed, AC is highly anisotropic mainly because of collagen fibres aligned in specific directions within the AC thickness. The modelling of AC full anisotropy is a challenging numerical task. However, as stated by Donzelli et al. (1999), the biphasic transversely isotropic non linear (BTINL) model is suitable to capture the AC anisotropy without explicitly accounting for the collagen network. A transversely isotropic material can be defined as a special case of an orthotropic material; namely, its mechanical properties are invariant with respect to rotations about an axis, called axis of symmetry, which is normal to the plane of isotropy (Ting 1996). In AC, the plane of isotropy is thought to be aligned with the superficial collagen fibrils, which run parallel the $A C$ surface.This assumption allows the stresses in the tissue can to be obtained by manipulating the elastic stiffness matrix to account for the planar isotropy. By invoking symmetries, it can be shown that only five independent material parameters are required in order to characterize the model (see Section 2.6).

This model, as shown using direct comparison with experimental data in (DiSilvestro, Zhu and Suh 2001), is capable of capturing both lateral displacements and reaction forces produced by an unconfined compression test, but it does not capture the evolution of these two variable simultaneously. This is probably caused by the absence of the viscoelasticity of the solid matrix.

\subsection{Material parameters}

In summary, five constitutive models of $A C$ were used in the present study: $M, B L, B N L, B P V N L$ and the BTINL. It should be noted that the non linearity (hence the acronym ending in NL) included in the BNL, BPVNL and BTINL models refers to the fluid non linear behaviour captured by using the permeability law given in Eq. (1). Furthermore three BPVNL models, characterized by different relaxation functions (BPVNLa, BPVNLb, and BPVNLc), were implemented in order to investigate the role of the viscoelasticity of the solid phase on the AC mechanical response. Collagen fibrils oriented parallel to the AC surface, i.e. running in $x$ and $z$ directions, were considered in the BTINL model.

The mechanical properties characterising each model (see Tables 1-3) were extracted from experimental studies reported in the literature and used to calibrate the parameters to be adopted for numerical implementation. For the monophasic model, $E_{\text {ins }}=10 \mathrm{MPa}$ and $v_{\text {ins }}=0.46$ were chosen from the ranges of values reported in literature, i.e. 4-20 $\mathrm{MPa}$ and 0.45-0.48, respectively (Bei and Fregly 2004, Beillas et al. 2004, Bendjaballah et al. 1997, Bendjaballah et al. 1995, Donahue et al. 2002, Donahue et al. 2003, Papaioannou et al. 2008, Pena et al. 2008, Pena et al. 2005). The parameters of the biphasic models BL, BNL, BPVNLa, BTINL, were taken from (DiSilvestro, Zhu, Wong et al. 2001). This allowed us to use a consistent set of parameters, i.e. derived from the same AC samples set, for different AC models and consequently a direct and rigorous comparison between the implemented models. It should be noted that the parameters $M$ and $e_{0}$ were not reported in (DiSilvestro, Zhu, Wong et al. 2001) and consequently they were estimated from other literature sources (Han et al. 2005, Pawaskar et al. 2010, Suh and Bai 1998, Wilson et al. 2003). The properties of the BPVNLb and BPVNLc models were taken from (DiSilvestro and Suh 2001) and (Suh and Bai 1998), respectively, assuming the same values of $M$ and $e_{0}$ used for the other models. 
All the AC constitutive models were implemented in the simplified TFJ FE model, whilst only the monophasic and the BPVNLb models were adopted in the patient specific TFJ model, as explained in Section 5. The FE models were developed using the FE package Abaqus 6.9, using predefined material models.

\section{Three dimensional simplified FE model of the TFJ}

\subsection{Model description}

As a first step towards the implementation of an advanced AC material model in realistic 3D knee geometries, a 3D simplified FE model of the TFJ has been developed, reproducing the realistic local anatomy of the joint. Simulations are therefore performed with the aim of identifying the optimal mesh and the most reliable constitutive law for AC to then be implemented in the patient specific FE model presented in Section 4. The simplified three-dimensional model of the TFJ depicted in Figure 1(a) is geometrically representative of a single knee compartment and is constituted by two interacting spherical layers of $A C$ bonded to two prismatic substrates representing the equivalent bone structures. An average thickness of the AC layers of $2.5 \mathrm{~mm}$ (Li et al. 2005, Liu et al. 2010) and curvature radius of $200 \mathrm{~mm}$ were selected as they correspond to the equivalent radius of curvature and cartilage thickness observed in the most heavily loaded TFJ contact regions. The bone is represented by a prismatic part characterised by a square base (16 $\mathrm{mm} \times 16$ $\mathrm{mm}$ ) and height $25 \mathrm{~mm}$. Only a quarter of the model was simulated since symmetric kinematic BCs were invoked (Section 3.3).

\subsection{Mesh}

Hexahedral elements were employed to mesh both the $A C$ and the bone. Different element types were used for each tissues/models: linear 3D Stress (C3D8R) for bone, quadratic 3D Stress (C3D20R) for monophasic AC, and quadratic Pore Fluid/Stress (C3D20RP) for biphasic AC. It is worth noting that hexahedral elements are a requirement for Soils Consolidation analyses performed by Abaqus; in addition, the quadratic order used for the AC elements was chosen in order to achieve better accuracy and avoid convergence issues. This is in accordance with the mesh sensitivity study performed in the present work (here not detailed for brevity); a thorough investigation tackling convergence and accuracy issues was also carried out to select the element size required to correctly capture the AC behaviour. On the basis of such analysis, which showed that the results are relatively insensitive to the choice of prismatic element base size, two mesh densities were selected: their topologies are depicted in Figure 1(b), with the finer one employed in the simplified 3D model to maximize accuracy and the coarser one used in the full 3D knee joint model to achieve an optimal compromise between accuracy and computational $\operatorname{cost}^{\ddagger}$.

\subsection{Boundary Conditions}

Surface interactions: Two different surface interactions occur in the TFJ: the bone-AC and the AC-AC interactions (Figure 2(a)). The bone-AC interaction was modelled as a tie constraint (i.e. no relative motion). This was thought to be a reasonable assumption since the $A C$ surface is strongly bonded to the correspondent bone surface where the AC tidemark lies. The AC-AC interaction was modelled as a frictionless (considering only normal stresses at the contact surface) and hard contact (the contact is "total" when it is detected), taking into account the possibility of finite sliding between the surfaces.

Kinematic and loading conditions: The lower transversal section of the tibial bone was built-in while the femur kinematics was driven by the kinematics of a reference point (RP) linked to the top rigid surface, as depicted in Figure 2(a). The vertical translation of the RP was left as unconstrained since this degree of freedom (DoF) was indirectly controlled by the vertical load; all the other DoFs were fixed. The loading cycle consisted in a vertical load of $10 \mathrm{~N}$ applied to the rigid surface using a ramp of $15 \mathrm{~s}$, then kept constant for $450 \mathrm{~s}$ (Figure 2(b)), which results in a maximum load of $80 \mathrm{~N}$ applied to the entire knee. Although such conditions do not reproduce the physiological conditions later adopted for the patient specific TFJ model, they were chosen for a number of reasons. First of all, the loading protocol simulated conditions similar to those of mechanical tests carried out to extrapolate AC material properties used in this study (Table 3): e.g. DiSilvestro and co-workers (DiSilvestro, Zhu, Wong et al. 2001) prescribed a strain rate of $0.001 \mathrm{~s}^{-}$ ${ }^{1}$ whilst here (see Section 5.1) the loading conditions resulted in a strain rate in the range $0.002-0.007 \mathrm{~s}^{-1}$ at the end of

₹ The convergence and sensitivity studies performed using the simplified 3D model showed that structured meshes characterized by at least eight "layers" of elements are required in order to accurately capture the transient response of the tissue when subjected to rapidly varying loads. However, increasing the number of elements used to discretise the AC in the TFJ results in a significant increase in computational time. A good compromise in terms of accuracy and computational time can be reached using four layers of elements to model the articular cartilage tissues in the full 3D joint model. It should also be noted that this discretisation strategy is similar to the one proposed by $\mathrm{Gu}$ and $\mathrm{Li}$ (2011) and Kazemi et al. (2011), although no information about mesh convergence and sensitivity analyses are reported in their contribution. 
the load ramp. Secondly, the simulation of low loads allowed to compare AC constitutive models and to gain insights into the transient mechanical response of $A C$ avoiding numerical difficulties and high computational costs; a similar approach has also been adopted in other studies presented in the recent literature (Gu and Li 2011).

BCs for the pore pressure of the biphasic AC: When the AC is modelled as a biphasic material, pore pressure BCs are implemented to regulate the fluid flow in and out of the tissue. As indicated in (Figure 2(c)), a sealed condition (i.e. no fluid flow) at the bone-AC interfaces, and a free draining condition (i.e. zero pore pressure) on the external $A C$ surfaces were employed.

\section{Patient specific FE model of the TFJ}

\subsection{Model description}

In this study, a bicompartmental total meniscectomised knee joint has been modelled with the aim to investigate the development of secondary OA. Consequently the anatomical structures of interest are the femur/tibia bone, the AC, the ligaments and the muscles. As the stabilising and the load transfer functions of ligament and muscles were reproduced by ad-hoc BCs, the patient specific model consists of the femur/tibia bone extremities and AC, which is modelled as a surface layer perfectly bonded to the bone.

The model geometry was obtained from high resolution medical images of a 75 years old female patient, with a body weight of $65 \mathrm{Kg}$, which had previously undergone a bicompartmental total meniscectomy surgery. The hard and soft tissue reconstructions were based on CT and MRI scans, respectively (Figure 3(a)). The 2D scans were later combined for the surface rendering by means of the AVIZO package.

The final model consists of six deformable parts, as shown in Figure 3(b): the two articulating bone extremities of the femur and the tibia, the lateral femoral AC (LFAC), the medial femoral AC (MFAC), the lateral tibial AC (LTAC), and the medial tibial AC (MTAC). A rigid body, corresponding to the upper transversal slice of the femur (coloured in grey in Figure 3(c)), was also included to enable the application of uniform loads on the upper bone cross-section.

\subsection{Mesh}

The reconstructed geometry was imported in Abaqus where all remaining phases of pre-processing, solving and post-processing of FE analysis were executed.

The geometric parts were individually meshed as portrayed in Figure 3(c). The mesh characteristics are detailed in Table 4. The bone and the AC were meshed with tetrahedral and hexahedral elements respectively (see Section 3.2). The dimension of the bone elements varied along the $Y$-axis, with the elements progressively reducing in size in the regions adjacent the contact region. The mesh of the AC sections was chosen according to the mesh sensitivity analysis performed using the simplified model described in Section 3, in order to guarantee both accuracy and low computational cost. Thus, 4 layers of prismatic elements of varying thickness and with a square base characterised by edge dimensions of about 1.5-2.5 mm were adopted for the discretisation of the AC tissue. It is worth noting that this mesh refinement was exploited also in other literature studies on 3D FEM knee modeling (Gu and Li 2011, Kazemi et al. 2011).

\subsection{Boundary conditions}

Surface interactions: The surface interactions were modelled as described in Section 3.3. The resulting model was composed of 4 pairs of tied surfaces at the AC-bone interfaces, and two contact pairs, in the medial (MFACMTAC) and in the lateral (LFAC-LTAC) compartments (Figure 4(a)).

Kinematic and loading conditions: The kinematic and loading BCs were chosen in order to simulate the adaptation mechanism of the articulating surfaces approaching contact, therefore allowing the surfaces to reach a relative position that ensures maximum contact area and minimum contact pressure. The lower transversal section of the tibia was built-in, while the femur kinematics was driven by the kinematics of the femoral rigid part, whose reference point (RP) was positioned between the two femoral condyles (Figure 4(a)). As for the simplified TFJ model, the vertical translation of the RP was controlled via the vertical load. The abduction/adduction, i.e. the rotation of the rigid part of the femur around the Z-axis, was considered free whilst all the other DoFs were fixed. The full-extension of the TFJ was chosen as the initial condition since this is considered one of the most critical loading conditions. All simulations were executed in load control. A physiological vertical load was applied to the femoral rigid part and transmitted, as a homogenously distributed pressure, to the upper transversal section of the femoral deformable part. The loading cycle, which was repeatedly applied to the joint for 5 times during the simulations, is illustrated in Figure 4(b). This corresponds to a simplified representation of the vertical load evolution observed during a gait cycle, which is characterised by a double peak during the stance phase and a constant low value during the swing phase. This is in 
agreement with both the predictions of other musculoskeletal models (Hurwitz et al. 1998, Shelburne et al. 2006) and the measurements carried out on instrumented knee prosthesis (Zhao et al. 2007). However, the peak load, which can reach up to $2.5 \mathrm{BW}$ during the gait, was here set to $1.5 \mathrm{BW}$, which corresponds to the maximum force during the gait with the knee in full extension (Fox and Delp 2010, Lamoreux 1971) and is also in line with the loading protocol used in the experimental tests (Fukubayashi and Kurosawa 1980) and in other FE models (Bendjaballah et al. 1995, Donahue et al. 2002, Mononen et al. 2012, Pena et al. 2006, Pena et al. 2005). It is worth mentioning that the load control can be implemented only when the joint surfaces are in contact. This condition was guaranteed by an initial phase of displacement control which consisted of a very small linear vertical displacement, applied in $0.1 \mathrm{~s}$.

BCs for the pore pressure of the biphasic AC: As already described in Section 3.3, sealed and free-draining conditions were imposed at the bone-AC interfaces and on the external AC surfaces, respectively. In this way we assumed that the interstitial fluid could exude out the AC across the free surfaces, as illustrated by the fluid velocity vectors computed during the simulation and shown in Figure 4(c).

\section{Results and discussion}

\subsection{Comparison of AC material models}

This section presents the comparison of the constitutive models of the AC implemented in the simplified TFJ model. Both global (i.e. vertical displacement of the RP and contact area) and local parameters ${ }^{\S}$ (e.g. stresses, strains, pore pressure, flow velocity) were monitored for the AC and the implications that their variations had on the tissue's response were investigated.

Initially the difference between the monophasic and the biphasic AC models was considered. The results reported in Figure 5 demonstrate that the monophasic material, whose properties have been calibrated as described in Section 2.6, seems to be stiffer than the biphasic model, being characterized by a lower vertical displacement of the RP (i.e. indication of soft tissue compression), a lower contact area and a higher contact pressure. The maximum mismatch between the models in terms of contact area and contact pressure are $85 \%$ and $450 \%$ respectively, as shown in Figures 5(b)-(c). Direct evidence of the fact that biphasic AC models are required to predict the realistic transient behaviour of the soft tissue is provided by the constant values obtained for both local and global characteristics when looking at all the outputs of the monophasic model displayed in Figure 5 after the load reaches the plateau (see Figure 2(b)). This is obviously not the case for the biphasic model, whereby the load support is generated by both the solid matrix and the fluid phase (Sakai et al. 2012) and their continuous interaction results in a time dependent material response (Figure 5). In particular, the ratio of the fluid/solid support varies both in time and in space within the AC thickness. This is clearly captured in Figure 6, which portrays the normalised solid and fluid support components along two node paths extracted under the surface of the bottom AC. The simulations, obtained assuming the BPVNLb model for the AC, show that, when the load is initially ramped to its maximum value, the load is almost entirely supported by the increase in interstitial fluid pressure in the tissue, which develops due to the very low permeability of AC; gradually, as the fluid exudes out of the contact zone, a load shift is observed from the fluid to the solid phase. It is worth noting that the solid and fluid supports were calculated as the integral of the vertical axial stress and the pore pressure along the path of nodes, respectively, and were normalised with respect to the total load.

We turn now to the differences between the various biphasic AC models considered in this study. Among the implemented BPVNL models, only the BPVNLa is considered for the purpose of comparison. As shown by the vertical displacement of the RP in Figure 5(a), during the monotonic application of the load the compression of the tissue increases, more or less linearly, depending on the adopted biphasic model; after the load is held constant at its maximum value, the vertical displacement keeps increasing for all models due to the fluid exudation and, in the case of the BPVNLa, also due to the viscoelastic relaxation of the ECM. The comparison between the responses obtained using different biphasic $A C$ models revealed that the compression of the tissue is largest for the BL model and smallest for the BPVNLa model, with the BNL and the BTINL producing intermediate results. This is due to the fact that the BNL model is stiffer than the $\mathrm{BL}$ model because of a lower fluid exudation due to the reduction of permeability as a function of the strains experienced by the solid matrix; furthermore, the BTINL material has a stiffness in the transverse direction higher than the BNL model. Moreover, with respect to all the other models, the matrix of the BPVNLa material is characterised by an additional stiffening due to the viscoelastic behaviour of the solid phase; such effect depends on the strain rate and is partly responsible for the behaviour displayed in Figure 5(a). The compression of the AC is directly related to the contact area and the contact pressure, depicted in Figures 5(b) and (c) respectively: the higher the strain, the better the adaptation of the articulating surfaces and, thus, the larger the contact area and the lower the

$\S$ A local parameter of a geometric part corresponds to the value of the variable of interest extrapolated at the node/element at which the maximum absolute value was recorded during the loading cycle. If the maximum value was shared by more nodes/elements, the variable was evaluated by averaging over these nodes/elements. 
contact pressure. Consequently, the lowest contact area and the highest contact pressure were observed for the BPVNLa model, whilst the highest contact area and the lowest contact pressure for the BL model. It is worth noting the significant dispersion of the local/global parameters predicted by the various biphasic models. For instance, a relative mismatch of $45.2 \%$ was found at the end of the loading cycle between the largest (BL model) and the smallest (BPVNLa model) contact area and a relative variation of about $54.5 \%$ was computed between the largest (BPVNLa model) and the smallest (BL model) peak contact pressure. The same data dispersion was observed for the pore (i.e. fluid) pressures of the biphasic models, compared in Figure 5(d). All models clearly show pore fluid pressurization as the load is applied; in particular the highest pore pressure was observed in the BPVNLa model, whilst the lowest in the $\mathrm{BL}$ model. When the load is kept constant at its maximum value, the increase in fluid exuded from the contact zone corresponds to a drastic decrease of the fluid pressure and an increase of the strain in the solid matrix.

Our results pointed out that the flow dependent viscoelasticity of biphasic AC enables more conformal contacts and hence allows the load to be distributed over a larger area, leading to lower contact pressure and stresses in the soft tissue. Also the flow independent viscoelasticity of BPVNL models is a fundamental aspect of AC behaviour: for high strain rates due to rapidly varying dynamic loading, it enables the stiffening of the soft tissue thus inhibiting excessive deformations, in agreement with Murakami et al. (2003). The influential role of the flow independent viscoelasticity is also highlighted by means of the comparison between the mechanical responses of several BPVNL models. Indeed BPVNLa-c models predicted quite different values of peak contact pressure (Figure 5(c)) and peak pore pressure (Figure 5(d)), and also different rates of stress relaxation, all in agreement with their viscoelastic parameters reported in Table 3. For instance the BPVNLb model predicted higher contact pressures when compared to BPVNLa and BPVNLc, with a discrepancy of up to $17.8 \%$ and $56 \%$, respectively.

This study has provided further evidence of the need to implement biphasic AC models; in particular, we have highlighted that using biphasic constitutive descriptions to model physiological loading conditions in real TFJ contact geometries allows predicting the realistic transient mechanical behaviour of the tissues under investigation, the load shift mechanism and the adaptation of the contacting surfaces together with the load redistribution. Although several TFJ contact studies have made use of the monophasic elastic model, relying on the fact that at high strain rates a biphasic material would behave as a monophasic model with a higher instantaneous elastic modulus, the implementation of biphasic constitutive models, even in their simplest form, is certainly a first step for achieving more accurate predictions of the stress state and the onset of damage of $A C$ as this is bound to be linked to the time-varying response of the tissue under impact loading. The BPVNL model was selected among the previously discussed biphasic $\mathrm{AC}$ models to be implemented in the patient specific TFJ model as it is the only one that accounts for the flow independent viscoelasticity and the predicted mechanical response shows very good agreement with several experimental findings obtained from different experimental configurations (e.g. confined/unconfined tests) (DiSilvestro and Suh 2001, DiSilvestro, Zhu and Suh 2001, DiSilvestro, Zhu, Wong et al. 2001).

\subsection{AC mechanical response during simplified walking cycles}

This section presents the main results obtained by simulating the patient specific TFJ contact under simplified walking cycles. The comparison between the outputs obtained using the monophasic (M) and one of the implemented biphasic models (i.e. BPVNLb) was considered to shed light on the AC behaviour. The main findings are reported in Figures 7-10, which show the results obtained by the two models in terms of both global and local responses ${ }^{\S}$ of the AC (Figure 7), also making use of relevant 3D contour plots (Figures 8-10) to aid the discussion.

\subsubsection{Monophasic vs BPVNL AC behaviour during walking: implications for the onset of damage and OA}

The comparison between monophasic and biphasic AC response during walking confirms the necessity to implement advanced $A C$ models in order to predict a realistic transient tissue response. During each walking cycle, the mechanical response of both the monophasic and the biphasic AC reflects the transient behaviour of the load: all the curves in Figure 7 are characterised by two peaks which correspond to the two loading peaks. Before each loading peak, the compressive strains (Figure 7(a)), the contact area (Figure 7(b)) and the contact pressure (Figure 7(c)) rapidly increase. In the model characterised by biphasic AC, an increase in the interstitial fluid pressure (Figure 7(d)) and of the magnitude of flow velocity is recorded, whilst both the void ratio and the permeability decrease. The opposite occurs as the load decreases after each loading peak. In particular, the fluid pressure remains always positive; this suggests a continuous fluid exudation (fluid flowing out of the tissue), in accordance with the free draining BC applied to the AC surface.

The differences between the two models appeared to be remarkable: the biphasic AC model predicted a temporal evolution of the mechanical response of the tissue, both within a single and several walking cycles, highlighting the unique capacity of the biphasic material to adapt to the load and to redistribute stresses and strains. Figures 7-9 show a significant variation of the biphasic $A C$ response during the first three cycles, and less variation in the last two cycles. Indeed, the AC behaviour approached a pseudo-steady state after about five walking cycles, when most of the fluid 
had exuded and the elastic response of the solid matrix dominates the tissue's behaviour. The value of the pore pressure in the MFAC decreased by $24.2 \%$ between the first peak load of the first and the third walking cycle, and by $7.5 \%$ between the first peak load of the third and the fifth walking cycle; the corresponding percentage decrements of the Tresca stress were $23.8 \% 21.1 \%$ and $7.9 \%, 6.8 \%$ respectively. In brief, the compression of the soft tissue continuously increased (Figure 7(a)) and allowed a better adaptation of the contacting surfaces. This corresponds to increasing contact areas (Figure 7(b)) and decreasing contact pressures (Figure 7(c)). Between the first peak load of the first and the last walking cycle, the variations of the contact pressure and the contact area in the medial side were $25.8 \%$ and $34.3 \%$ respectively. The load, at first mainly supported by the fluid pressurisation, was gradually transferred to the solid matrix as the fluid exuded the pore pressure decreased (Figure 7(d)); furthermore, the Tresca stresses decreased as a consequence of the poroviscoelastic stress relaxation (Figure 9). The stress and strains redistribution that characterizes the behaviour of the biphasic AC is clearly described by the contour plots in Figures 8-10: during the cyclic application of the load both the Tresca stress and the contact pressure decrease, while the contact area increases.

It can be concluded that the longer one walks, the less stressed the tibio-femoral AC is going to be! A mechanism of load redistribution in the tissue occurs which is fundamental to prevent the damage of the solid constituents. During the initial loading phase of walking, the tissue is subject to the highest stresses. After just a few walking cycles, the AC adapts to the loading cycle and shows a quasi-steady behaviour characterised by higher contact area and lower stresses. The limits of the monophasic AC model are thus highlighted: it fails to account for the load redistribution and it tends to overestimate the contact area and underestimate the maximum instantaneous stresses.

It should be borne in mind, however, that the stress decrease observed in this study might not necessarily occur in every TFJ configuration and loading scenario. This is a complex phenomenon dictated by the interplay between the variation of interstitial fluid support and the change in stresses due to the adaptation of the joint. The balance between the two, which ultimately dictates the overall resulting stress levels, depends upon the contact geometry and loading conditions which would vary from joint to joint.

The model presented in this work to study the mechanical response of a full knee joint suffering from a total bicompartmental meniscectomy is motivated by the will to investigate the initiation of AC damage and OA associated with such a procedure (Korkala et al. 1984, Lanzer and Komenda 1990). Indeed, because of the absence of the menisci, the biomechanics of a healthy knee is completely modified and high stresses can be experienced by the AC, therefore promoting tissue damage (McDermott and Amis 2006). Some studies have addressed the damage initiation in AC using FEM (Askew and Mow 1978, Eberhardt et al. 1991, Garcia et al. 1998, Kelly and O'Connor 1996); however, researchers have not yet investigated the damage initiation in a patient specific and anatomically realistic TFJ contact both using advanced AC models and in the presence of physiological loading conditions. Investigating damage initiation goes beyond the aim of the present study; however, some preliminary considerations on this issue will be reported here to discuss our initial efforts in what will constitute the focus of our future investigations.

Most FE models of the knee joint, which attempt to predict AC damage initiation, typically investigate maximum shear stress (Eberhardt et al. 1991, Garcia et al. 1998), Tresca stress distributions, maximum principal strains (Askew and Mow 1978) in the $A C$ and identify regions with the highest stresses as being the locations more prone to $O A$ initiation and damage. Furthermore, a new criterion was recently introduced by Pena et al. (2008) based on the hypothesis that the $A C$ failure is mainly due to the local variation of shear stresses rather than their absolute magnitude; this has shown some promise and the predictions were somewhat in agreement with existing experimental studies. The exploitation of classical von Mises and Tresca failure criteria has certainly some limitations considering that they were first established for ordinary engineering materials which clearly have a completely different mechanical behaviour compared to the biphasic AC, and cannot account for the mechanisms that cause AC damage at the microstructural level. Nevertheless, from a macroscopic point of view, it is reasonable to expect that high local stresses will correspond to the most damaged areas (Wilson et al. 2006). Since the aim of or investigation is to compare AC damage predicted using monophasic and biphasic constitutive behaviours, in the present study we simply analysed locations of $\mathrm{OA}$ initiation suggested by the maximum of von Mises and Tresca stresses. As such locations were predicted to be almost identical according to the two measures of stress, only the results relative to the Tresca stress are here presented and discussed. As expected, the biphasic and the monophasic constitutive laws predicted a different stress field in the soft tissue, both in terms of magnitude and location (Figure 10). First of all the two models predicted the highest Tresca stresses in different TFJ compartments, i.e. in the MFAC and in the LFAC for the BPVNL and the $\mathrm{M}$ model respectively, although both the models simulated a more conformal contact and a wider contact area in the medial compartment. Secondly, a different distribution of Tresca stresses was also observed in the AC thickness. Figure 10(a) shows the map of Tresca stresses simulated by the BPVNL models at the first load peak in a section of the medial $A C$ compartment belonging to the frontal plane and crossing the point of the maximum superficial Tresca stress. The Tresca stresses for the AC is maximum at the AC surface during the entire duration of the simulations, almost at the same locations as the maximum contact pressure, while decrease through the AC depth. However, it should be considered that the maximum Tresca stress varies in magnitude and location with time (see also Figure 9). 
On the other hand, the monophasic model predicted much lower Tresca stresses in the same AC section and at the same instant (Figure 10(b)) and, in contrast to what is seen for the BPVNL model, the highest Tresca stresses are approximately at the centre of the AC thickness.

In conclusion, according to the biphasic constitutive law and assuming a conventional macroscopic failure criterion, e.g. looking for the maximum Tresca stresses as an indicator of damage, and thus considering the evidence that shear stresses are responsible for the collagen fibres failure and the initiation of tissue degeneration, the most likely location for the OA initiation in the bicompartmental meniscectomised knee was predicted to be on the medial AC surface. In particular, by analysing the superficial Tresca stresses distribution (Figures 9-10(a)), OA could initiate in the region near the centre for the medial compartment and in the proximity of the $\mathrm{AC}$ surface edge for the lateral compartment, almost in correspondence of the maximum contact pressures. This is in line with previous studies and clinical data which have showed that $O A$ affects the medial compartment significantly more than the lateral compartment (Ahlback 1968, Hernborg and Nilsson 1977, White et al. 1991). Furthermore, the central aspect of the compartment seems to be more prone to damage compared to the anterior and posterior aspects(Andriacchi et al. 2004).

\subsubsection{Model validation using experimental evidence and comparison with existing numerical simulations}

In the past decades several experimental studies on cadaveric/amputated human knees have been carried out to investigate the effects of meniscectomy on knee contact mechanics in terms of contact area and contact pressure, generally measured using flexible pressure-sensitive films (e.g. Prescale by Fuji Film Co. (Fukubayashi and Kurosawa 1980)). A comparison with these results can be difficult since experimental tests can differ in BCs or be affected by inter-subject variability. The closest match to the scenario modelled in this paper is provided by the experimental results obtained by Fukubayashi and Kurosawa (1980). Under a compression load of $1000 \mathrm{~N}$, with the knee in full extension, they measured, for fully meniscectomised knees, a contact area of about $300 \pm 80 \mathrm{~mm}^{2}$ in the medial compartment and $230 \pm 80 \mathrm{~mm}^{2}$ in the lateral one, and a peak contact pressure around $6 \mathrm{MPa}$. These results are in good agreement with our simulations, thus corroborating the results obtained using a biphasic description of the tissue: if $1000 \mathrm{~N}$ are applied to the BPVNL model, the predicted contact areas are in the range of 203-282 $\mathrm{mm}^{2}$ and $146-182$ $\mathrm{mm}^{2}$, for the medial and the lateral side respectively, while the peak contact pressure is in the range of $4.5-6.3 \mathrm{MPa}$. In addition, our results showed larger contact areas in the medial compartment, as also observed in several ex-vivo studies of total meniscectomised knees (Fukubayashi and Kurosawa 1980, Ihn et al. 1993).

A comparison with existing numerical studies can be even harder considering the wide variety of geometries, BCs and AC material laws/properties simulated in the recent contributions presented in the literature. However, an attempt is made here to compare our results with some of the outputs obtained by other researchers using models of fullyextended knee under compression axial loads. First, FE simulations performed assuming the AC as an elastic isotropic material are compared to our results obtained using monophasic AC model. It is worth noting that such models simulated total medial meniscectomised knee and, thus, only results relative to the medial compartment are considered here. Bendjaballah et al. (1995) predicted, under $1000 \mathrm{~N}$ load, compressive stresses of about $2.8 \mathrm{MPa}$ and $5 \mathrm{MPa}$ in MTAC and MFAC respectively; for a higher load of $1150 \mathrm{~N}$, Pena et al. (2005) computed compressive stresses of 5.3 MPa and 6.4 MPa in MTAC and MFAC respectively. Again for a compressive load of $1150 \mathrm{~N}$, Pena et al. (2008), when considering the Tresca stresses in order to predict the onset to OA, obtained maximum values for this parameter of $6 \mathrm{MPa}$ and 3.3 MPa in MTAC and MFAC respectively. If a direct comparison with our model is sought, the results obtained for the monophasic $A C$ description in our simulations at $1000 \mathrm{~N}$ show maximum compressive stresses of 3.9 MPa and 4.1 MPa in MTAC and MFAC respectively and maximum Tresca stress of about 2.7 MPa. According to (Bae et al. 2012), simulations carried out using a load of $570 \mathrm{~N}$ show a peak contact pressure of $3.3 \mathrm{MPa}$ and $3 \mathrm{MPa}$ in MTAC and MFAC respectively, while the values obtained using the model presented here gives $3.5 \mathrm{MPa}$ and $2.9 \mathrm{MPa}$ respectively. To conclude, the TFJ model proposed in the present study and produced by the authors assuming a monophasic description of the soft tissue is in good agreement with published studies.

Fewer studies on bicompartmental total meniscectomy have been carried out implementing biphasic description of AC. Wilson et al. (2003) proposed a simplified axysimmetric knee model with biphasic AC layers: under a load of 588 $\mathrm{N}$, applied in $1 \mathrm{~s}$, a maximum contact pressure of $1.5 \mathrm{MPa}$ for BNLE model and 1.9 MPa for a BTINLE model were obtained. Under the same load, but applied at higher load rates, our model predicts, as expected, a higher maximum contact pressure of about 4.7 MPa. More recently, a 3D knee model with fibril reinforced biphasic AC was developed by Kazemi et al. (2011); a load ramp of $300 \mathrm{~N}$ in $1 \mathrm{~s}$ caused a maximum principal stress of $0.52 \mathrm{MPa}$ and a maximum fluid pressure of $0.6 \mathrm{MPa}$, both in a deep layer of MFAC that is approximately at $7 / 8$ depth from the AC surface. The results obtained using the proposed biphasic description for a load of $300 \mathrm{~N}$ applied in $0.11 \mathrm{~s}$ were $0.7 \mathrm{MPa}$ and 3 $\mathrm{MPa}$, respectively. As in our simulations the load rate was significantly higher than in (Kazemi et al. 2011) (e.g. about $3000 \mathrm{~N} / \mathrm{s}$ vs $300 \mathrm{~N} / \mathrm{s}$ ), the higher fluid pressurization level can explain the larger values of pressures and stresses predicted in our simulations. 


\section{Conclusion}

The present study highlights the importance of the implementation of advanced biphasic AC models to capture the fluid load support and the transient behaviour of the tissue response when modelling human joints. The prediction of damage initiation location in such joints can only be achieved by mimicking the mechanisms governing the behaviour of the tissue and by correctly reproducing the realistic stress and strain distributions within the AC.

In particular, the comparison of different biphasic constitutive laws performed on a simplified three-dimensional model of one of the TFJ compartments is used to show that both flow dependent and flow independent viscoelasticity are important and necessary to model the realistic mechanical response of the AC. The biphasic poroviscoelastic non linear description of the tissue has then been implemented in a patient specific joint model. The model is relatively simple to implement and captures the most important mechanisms governing the AC response under loading. In particular, it has allowed highlighting the load shift mechanism between the fluid/solid constituents of the tissue, studying the adaptation of $A C$ to the applied load with time, and identifying the most stressed areas of the $A C$, which in turn are most likely to be affected by OA (i.e. medial side). For the first time, a patient specific knee model has been developed to include and compare the effect that the monophasic and biphasic models of the AC have on the joint. The comparison between the results provided by this study and the existing simulations of meniscectomised joints have emphasised the need to explicitly model the biphasic nature of the AC. It is worth noting that the patient specific TFJ model has also been corroborated using experimental results obtained by studies carried out on cadaveric knees and available in the literature.

Ongoing studies are devoted to implement flow continuity across the AC tissues in three-dimensional joints, optimize the BCs in order to simulate more realistic walking conditions both in terms of load (e.g. torque) and kinematics (e.g. flexion, anterior-posterior translation), and include one or both menisci in the model in order to reproduce partial meniscectomy and healthy knees. The study of the interplay between the solid and fluid non linearities, the implementation of the 3D fibril-reinforced biphasic model to be tested under physiological loads and the development of a micromechanical damage model to be implemented in the current FE framework are also envisaged.

\section{Acknowledgments}

The authors wish to acknowledge Professor Justin P. Cobb, for his valuable contribution towards this study, the Imperial College High Performance Computing Service and Mahmut Tuncer for their precious support.

\section{References}

Accardi MA and Dini D. 2009. Modelling of the mechanical behaviour of human joints cartilage. In: STLE/ASME International Joint Tribology Conference 2008 New York: Amer Soc Mechanical Engineers. p. 147-149.

Ahlback S. 1968. Osteoarthrosis of the knee. A radiographic investigation. Acta Radiol Diagn (Stockh):Suppl 277:277-272.

Andriacchi TP, Mundermann A, Smith RL, Alexander EJ, Dyrby CO and Koo S. 2004. A framework for the in vivo pathomechanics of osteoarthritis at the knee. Annals of biomedical engineering. 32(3):447-457.

Appleyard RC, Burkhardt D, Ghosh P, Read R, Cake M, Swain MV and Murrell GAC. 2003. Topographical analysis of the structural, biochemical and dynamic biomechanical properties of cartilage in an ovine model of osteoarthritis. Osteoarthr Cartilage. 11(1):65-77.

Armstrong CG, Lai WM and Mow VC. 1984. An Analysis of the Unconfined Compression of Articular Cartilage. J Biomech Eng. 106(2):165-173.

Askew MJ and Mow VC. 1978. Biomechanical Function of Collagen Fibril Ultrastructure of Articular-Cartilage. J Biomech Eng. 100(3):105-115.

Bae J, Park K, Seon J, Kwak D, Jeon I and Song E. 2012. Biomechanical analysis of the effects of medial meniscectomy on degenerative osteoarthritis. Med Biol Eng Comput. 50(1):53-60.

Baratz ME, Fu FH and Mengato R. 1986. Meniscal tears: the effect of meniscectomy and of repair on intraarticular contact areas and stress in the human knee. A preliminary report. Am J Sports Med. 14(4):270-275.

Bei Y and Fregly BJ. 2004. Multibody dynamic simulation of knee contact mechanics. Med Eng Phys. 26(9):777-789. 
Beillas P, Papaioannou G, Tashman S and Yang KH. 2004. A new method to investigate in vivo knee behavior using a finite element model of the lower limb. J Biomech. 37(7):1019-1030.

Bendjaballah MZ, Shepherd DET and Zukor DJ. 1997. Finite element analysis of human knee joint in varus-valgus. Clin Biomech. 12(3):139-148.

Bendjaballah MZ, Shirazi-AdI A and Zukor DJ. 1995. Biomechanics of the human knee joint in compression: reconstruction, mesh generation and finite element analysis. Knee. 2:69-79.

Brown TD and Singerman RJ. 1986. Experimental-Determination of the Linear Biphasic Constitutive Coefficients of Human-Fetal Proximal Femoral Chondroepiphysis. J Biomech. 19(8):597-605.

Buckwalter JA and Mankin HJ. 1998. Articular cartilage: degeneration and osteoarthritis, repair, regeneration, and transplantation. Instr Course Lect. 47:487-504.

Buckwalter JA and Martin JA. 2006. Osteoarthritis. Adv Drug Deliv Rev. 58(2):150-167.

Crevoisier X, Munzinger U and Drobny T. 2001. Arthroscopic partial meniscectomy in patients over 70 years of age. Arthroscopy. 17(7):732-736.

DiSilvestro MR and Suh J-KF. 2001. A cross-validation of the biphasic poroviscoelastic model of articular cartilage in unconfined compression, indentation, and confined compression. $\mathrm{J}$ Biomech. 34(4):519-525.

DiSilvestro MR, Zhu Q and Suh J-KF. 2001. Biphasic poroviscoelastic simulation of the unconfined compression of articular cartilage: II - Effect of variable strain rates. J Biomech Eng. 123(2):198-200.

DiSilvestro MR, Zhu Q, Wong M, Jurvelin JS and Suh J-KF. 2001. Biphasic poroviscoelastic simulation of the unconfined compression of articular cartilage: I - Simultaneous prediction of reaction force and lateral displacement. J Biomech Eng. 123(2):191-197.

Donahue TLH, Hull ML, Rashid MM and Jacobs CR. 2002. A finite element model of the human knee joint for the study of tibio-femoral contact. J Biomech Eng. 124(3):273-280.

Donahue TLH, Hull ML, Rashid MM and Jacobs CR. 2003. How the stiffness of meniscal attachments and meniscal material properties affect tibio-femoral contact pressure computed using a validated finite element model of the human knee joint. J Biomech. 36(1):19-34.

Donzelli PS, Spilker RL, Ateshian GA and Mow VC. 1999. Contact analysis of biphasic transversely isotropic cartilage layers and correlations with tissue failure. J Biomech Eng. 32:1037 - 1047.

Eberhardt AW, Lewis JL and Keer LM. 1991. Contact of Layered Elastic Spheres as a Model of Joint Contact - Effect of Tangential Load and Friction. J Biomech Eng - Trans ASME. 113(1):107-108.

Englund M and Lohmander LS. 2004. Risk factors for symptomatic knee osteoarthritis fifteen to twenty-two years after meniscectomy. Arthritis Rheum. 50(9):2811-2819.

Fergusson CM. 1987. The aetiology of osteoarthritis. Postgrad Med J. 63(740):439-445.

Fox MD and Delp SL. 2010. Contributions of muscles and passive dynamics to swing initiation over a range of walking speeds. J Biomech. 43(8):1450-1455.

Fukubayashi T and Kurosawa H. 1980. The Contact Area and Pressure Distribution Pattern of the Knee: A Study of Normal and Osteoarthrotic Knee Joints. Acta Orthop. 51(1-6):871-879.

Fung YC. 1972. Stress - strain history relations of soft tissues in simple elongation. In: Biomechanics: Its Foundations and Objectives Englewood Cliffs, NJ: Prentice - Hall. p. 181 - 208.

Garcia JJ, Aliero NJ and Haut RC. 1998. An Approach for Stress Analysis of Trasnsversely Isotropic Biphasic Cartilage Under Impact Load. J Biomech Eng. 120:608 - 613.

Grodzinsky AJ, Roth V, Myers E, Grossman WD and Mow VC. 1981. The significance of electromechanical and osmotic forces in the nonequilibrium swelling behavior of articular cartilage in tension. J Biomech Eng. 103(4):221-231.

Gu KB and Li LP. 2011. A human knee joint model considering fluid pressure and fiber orientation in cartilages and menisci. Med Eng Phys. 33(4):497-503.

Guo Y, Zhang X and Chen W. 2009. Three-Dimensional Finite Element Simulation of Total Knee Joint in Gait Cycle. Acta Mech Solida Sin. 22(4):347-351.

Han S-K, Federico S, Epstein M and Herzog W. 2005. An articular cartilage contact model based on real surface geometry. J Biomech. 38(1):179-184.

Hede A, Larsen E and Sandberg H. 1992. The long term outcome of open total and partial meniscectomy related to the quantity and site of the meniscus removed. Int Orthop. 16(2):122-125.

Hernborg JS and Nilsson BE. 1977. The natural course of untreated osteoarthritis of the knee. Clin Orthop Relat Res(123):130-137. 
Holmes MH and Mow VC. 1990. The nonlinear characteristics of soft gels and hydrated connective tissues in ultrafiltration. J Biomech. 23(11):1145-1156.

Hosoda N, Sakai N, Sawae Y and Murakami T. 2010. Finite Element Analyses of Articular Cartilage Models Considering Depth-Dependent Elastic Modulus and Collagen Fiber Network. JBSE. 5(4):437-448.

Hurwitz DE, Sumner DR, Andriacchi TP and Sugar DA. 1998. Dynamic knee loads during gait predict proximal tibial bone distribution. J Biomech. 31(5):423-430.

Ihn JC, Kim SJ and Park IH. 1993. In vitro study of contact area and pressure distribution in the human knee after partial and total meniscectomy. Int Orthop. 17(4):214-218.

Julkunen P, Korhonen RK, Herzog W and Jurvelin JS. 2008. Uncertainties in indentation testing of articular cartilage: A fibril-reinforced poroviscoelastic study. Med Eng Phys. 30(4):506-515.

Kazemi M, Li LP, Savard P and Buschmann MD. 2011. Creep behavior of the intact and meniscectomy knee joints. J Mech Behav Biomed Mater. 4(7):1351-1358.

Kelly PA and O'Connor JJ. 1996. Transmission of Rapidly Applied Loads Through Articular Cartilage Part 1: Uncracked Cartilage. Proc Inst Mech Eng H J Eng Med. 210(1):27-37.

Knecht S, Vanwanseele B and Stussi E. 2006. A review on the mechanical quality of articular cartilage - implications for the diagnosis of osteoarthritis. Clin Biomech (Bristol, Avon). 21(10):999-1012.

Korkala O, Karaharju E, Gronblad M and Aalto K. 1984. Articular-Cartilage after Meniscectomy Rabbit Knees Studied with the Scanning Electron-Microscope. Acta Orthop Scand Suppl. 55(3):273-277.

Kurosawa H, Fukubayashi T and Nakajima H. 1980. Load-bearing mode of the knee joint: physical behavior of the knee joint with or without menisci. Clin Orthop Relat Res. 149:283-290.

Lai WM, Hou JS and Mow VC. 1991. A Triphasic Theory for the Swelling and Deformation Behaviors of Articular Cartilage. J Biomech Eng. 113(3):245-258.

Lai WM, Mow VC and Roth V. 1981. Effects of nonlinear strain-dependent permeability and rate of compression on the stress behavior of articular cartilage. J Biomech Eng. 103(2):61-66.

Lamoreux LW. 1971. Kinematic measurements in the study of human walking. Bull Prosthet Res. 10(15):3-84.

Lanzer WL and Komenda G. 1990. Changes in Articular-Cartilage after Meniscectomy. Clin Orthop Relat Res(252):41-48.

LeRoux MA, Arokoski J, Vail TP, Guilak F, Hyttinen MM, Kiviranta I and Setton LA. 2000. Simultaneous changes in the mechanical properties, quantitative collagen organization, and proteoglycan concentration of articular cartilage following canine meniscectomy. J Orthop Res. 18(3):383-392.

Li G, Park SE, DeFrate LE, Schutzer ME, Ji L, Gill TJ and Rubash HE. 2005. The cartilage thickness distribution in the tibiofemoral joint and its correlation with cartilage-to-cartilage contact. Clin Biomech. 20(7):736-744.

Li L, Cheung J and Herzog W. 2009. Three-dimensional fibril-reinforced finite element model of articular cartilage. Med Biol Eng Comput. 47(6):607-615.

Li LP and Gu KB. 2011. Reconsideration on the use of elastic models to predict the instantaneous load response of the knee joint. Proceedings of the Institution of Mechanical Engineers, Part H (Journal of Engineering in Medicine). 225(9):888-896.

Li LP, Shirazi-Adl A, Buschmann MD and Soulhat J. 1999. Nonlinear analysis of cartilage in unconfined ramp compression using a fibril reinforced poroelastic model. Clin Biomech. 14(9):673-682.

Liu F, Kozanek M, Hosseini A, Van de Velde SK, Gill TJ, Rubash HE and Li G. 2010. In vivo tibiofemoral cartilage deformation during the stance phase of gait. J Biomech. 43(4):658-665.

$\mathrm{Lu}$ XL, Wan LQ, Edward Guo X and Mow VC. 2010. A linearized formulation of triphasic mixture theory for articular cartilage, and its application to indentation analysis. J Biomech. 43(4):673-679.

Mak AF. 1986. The apparent viscoelastic behavior of articular cartilage-the contributions from the intrinsic matrix viscoelasticity and interstitial fluid flows. J Biomech Eng. 108(2):123-130.

McDermott ID and Amis AA. 2006. The consequences of meniscectomy. J Bone Joint Surg Br. 88(12):1549-1556.

McGinty JB, Geuss LF and Marvin RA. 1997. Partial or total meniscectomy: a comparative analysis. J Bone Joint Surg. 59(6):763-766.

McNicholas MJ, Rowley DI, McGurty D, Adalberth T, Abdon P, Lindstrand A and Lohmander LS. 2000. Total meniscectomy in adolescence: a thirthy-year follow up. J Bone Joint Surg. 82-B(2):217-221. 
Moglo KE and Shirazi-Adl A. 2005. Cruciate coupling and screw-home mechanism in passive knee joint during extension-flexion. J Biomech. 38(5):1075-1083.

Mononen M, Julkunen P, Töyräs J, Jurvelin J, Kiviranta I and Korhonen R. 2011. Alterations in structure and properties of collagen network of osteoarthritic and repaired cartilage modify knee joint stresses. Biomech Model Mechanobiol. 10(3):357-369.

Mononen ME, Mikkola MT, Julkunen P, Ojala R, Nieminen MT, Jurvelin JS and Korhonen RK. 2012. Effect of superficial collagen patterns and fibrillation of femoral articular cartilage on knee joint mechanics A 3D finite element analysis. J Biomech. 45(3):579-587.

Mow VC, Kuei S.C., Lai WM and Armstrong CG. 1980. Biphasic Creep and Stress Relaxation of Articular Cartilage in Compression: Theory and Experiments. J Biomech Eng. 102:73 - 84.

Murakami T, Ihara M and Sawae Y. 2003. Protective mechanism of articular cartilage to severe loading: roles of lubricants, cartilage surface layer, extracellular matrix and chondrocyte. JSME Int J Ser C. 46(2):594-603.

Oakley SP, Lassere MN, Portek I, Szomor Z, Ghosh P, Kirkham BW, Murrell GAC, Wulf S and Appleyard RC. 2004. Biomechanical, histologic and macroscopic assessment of articular cartilage in a sheep model of osteoarthritis. Osteoarthr Cartilage. 12(8):667-679.

Papaioannou G, Nianios G, Mitrogiannis C, Fyhrie D, Tashman S and Yang KH. 2008. Patientspecific knee joint finite element model validation with high-accuracy kinematics from biplane dynamic Roentgen stereogrammetric analysis. J Biomech. 41(12):2633-2638.

Papalia R, Del Buono A, Osti L, Denaro V and Maffulli N. 2011. Meniscectomy as a risk factor for knee osteoarthritis: a systematic review. British Medical Bulletin. 99(1):89-106.

Pawaskar SS, Fisher J and Jin ZM. 2010. Robust and general method for determining surface fluid flow boundary conditions in articular cartilage contact mechanics modeling. J Biomech Eng. 132(3):031001-031001.

Pena E, Calvo B, Martinez MA and Doblare M. 2008. Computer simulation of damage on distal femoral articular cartilage after meniscectomies. Comput Biol Med. 38(1):69-81.

Pena E, Calvo B, Martinez MA and Doblaré M. 2006. A three-dimensional finite element analysis of the combined behavior of ligaments and menisci in the healthy human knee joint. J Biomech. 39(9):1686-1701.

Pena E, Calvo B, Martínez MA, Palanca D and Doblaré M. 2005. Finite element analysis of the effect of meniscal tears and meniscectomies on human knee biomechanics. Clin Biomech. 20(5):498-507.

Petty CA and Lubowitz JH. 2010. Does Arthroscopic Partial Meniscectomy Result in Knee Osteoarthritis? A Systematic Review With a Minimum of 8 Years' Follow-up. Arthroscopy. 27(3):419-424.

Rieppo J, Hyttinen MM, Lappalainen R, Jurvelin JS and Helminen HJ. Spatial determination of water, collagen and proteoglycan contents by Fourier transform infrared imaging and digital densitometry. In. Transactions of the Orthopaedic Research Society.

Roos EM, Â-stenberg A, Roos H, Ekdahl C and Lohmander LS. 2001. Long-term outcome of meniscectomy: symptoms, function, and performance tests in patients with or without radiographic osteoarthritis compared to matched controls. Osteoarthr Cartilage. 9(4):316-324.

Sakai N, Hagihara Y, Furusawa T, Hosoda N, Sawae Y and Murakami T. 2012. Analysis of biphasic lubrication of articular cartilage loaded by cylindrical indenter. Tribol Int. 46(1):225-236. CRC Press.

Schneck DJ and Bronzino JD. 2002. Biomechanics : principles and applications. Boca Raton, FL:

Shelburne KB, Torry MR and Pandy MG. 2006. Contributions of muscles, ligaments, and the ground-reaction force to tibiofemoral joint loading during normal gait. J Orthop Res. 24(10):1983-1990.

Shirazi R and Shirazi-Adl A. 2009. Analysis of partial meniscectomy and ACL reconstruction in knee joint biomechanics under a combined loading. Clin Biomech. 24(9):755-761.

Song Y, Greve JM, Carter DR and Giori NJ. 2008. Meniscectomy alters the dynamic deformational behavior and cumulative strain of tibial articular cartilage in knee joints subjected to cyclic loads. Osteoarthr Cartilage. 16(12):1545-1554.

Soulhat J, Buschmann MD, Shirazi-Adl A and Li LP. 1999. Nonlinear analysis of cartilage in unconfined ramp compression using a fibril reinforced poroelastic model. Clin Biomech. 14(9):673-682.

Suh JK and Bai S. 1998. Finite Element Formulation of Biphasic Poroviscoelastic Model for Articular Cartilage. J Biomech Eng. 120:195 - 201. 
Ting TCT. 1996. Anisotropic Elasticity: Theory and Applications. New York: Oxford Engineering Science, Inc.

Torzilli PA and Mow VC. 1976a. On the fundamental fluid transport mechanisms through normal and pathological articular cartilage during function - I. The formulation. J Biomech. 9(8):541-552.

Torzilli PA and Mow VC. 1976b. On the fundamental fluid transport mechanisms through normal and pathological articular cartilage during function - II. The analysis, solution and conclusions. J Biomech. 9(9):587-606.

Van der Voet A. 1997. A comparison of finite element codes for the solution of biphasic poroelastic problems. Proc Inst Mech Eng H J Eng Med. 211(2):209-211.

White SH, Ludkowski PF and Goodfellow JW. 1991. Anteromedial osteoarthritis of the knee. J Bone Joint Surg. 73(4):582-586.

Wilson W, van Burken C, van Donkelaar C, Buma P, van Rietbergen B and Huiskes R. 2006. Causes of mechanically induced collagen damage in articular cartilage. J Orthop Res.

Wilson W, Van Donkelaar C, Van Rietbergen B and Huiskes R. 2005a. A fibril-reinforced poroviscoelastic swelling model for articular cartilage. J Biomech. 38(6):1195-1204.

Wilson W, van Donkelaar CC, van Rietbergen R and Huiskes R. 2005b. The role of computational models in the search for the mechanical behavior and damage mechanisms of articular cartilage. Med Eng Phys. 27(10):810-826.

Wilson W, Van Rietbergen B, Van Donkelaar CC and Huiskes R. 2003. Pathways of load-induced cartilage damage causing cartilage degeneration in the knee after meniscectomy. J Biomech. 36(6):845851.

Wu JZ and Herzog W. 2002. Simulating the swelling and deformation behaviour in soft tissues using a convective thermal analogy. BioMedical Engineering OnLine 2002. 1(8):1-11.

Yang NH, Canavan PK, Nayeb-Hashemi H, Najafi B and Vaziri A. 2010. Protocol for constructing subject-specific biomechanical models of knee joint. Comput Methods Biomech Biomed Engin. 13(5):589603.

Young A, McLennan S, Smith M, Smith S, Cake M, Read R, Melrose J, Sonnabend D, Flannery C and Little C. 2006. Proteoglycan 4 downregulation in a sheep meniscectomy model of early osteoarthritis. Arthrit Res Ther. 8(2):R41.

Zhao D, Banks SA, D'Lima DD, Colwell CW and Fregly BJ. 2007. In vivo medial and lateral tibial loads during dynamic and high flexion activities. J Orthop Res. 25(5):593-602. 


\section{List of tables}

Table 1. Mechanical parameters for the BL and the BNL models of AC (DiSilvestro, Zhu, Wong et al. 2001).

\begin{tabular}{|c|c|c|c|}
\hline \multirow{4}{*}{ BL } & $E_{e q}(\mathrm{MPa})$ & 0.421 \\
\hline \multirow{3}{*}{ BNL } & $v_{e q}$ & 0.28 \\
\hline & $k_{o}\left(\mathrm{~m}^{4} / \mathrm{N} \mathrm{s}\right)$ & $6.910^{-15}$ \\
\hline & ${ }^{*} \rho\left(\mathrm{g} / \mathrm{cm}^{3}\right)$ & 1 \\
\hline & ${ }^{*} e_{0}$ & 4 \\
\hline & ${ }^{*} M$ & 5 \\
\hline
\end{tabular}

Table 2. Mechanical parameters for the BPVNL models of AC (BPVNLa (DiSilvestro, Zhu, Wong et al. 2001), BPVNLb (DiSilvestro and Suh 2001), BPVNLc (Suh and Bai 1998)), in addition to the marked parameters (*) of Table 1.

\begin{tabular}{|c|c|c|c|c|}
\cline { 3 - 5 } \multicolumn{2}{c|}{} & BPVNLa & BPVNLb & BPVNLc \\
\hline \multirow{4}{*}{ BPVNL } & $E_{e q}(\mathrm{MPa})$ & 0.55 & 0.63 & 0.7 \\
\cline { 2 - 5 } & $v_{e q}$ & 0.24 & 0.1 & 0 \\
\cline { 2 - 5 } & $k_{0}\left(\mathrm{~m}^{4} / \mathrm{N} \mathrm{s}\right)$ & $0.9 \cdot 10^{-15}$ & $1.72 \cdot 10^{-15}$ & $2 \cdot 10^{-15}$ \\
\cline { 2 - 5 } & $\breve{G}$ & 15.180 & 17.692 & 10.21 \\
\cline { 2 - 5 } & $\tau_{S}(\mathrm{~s})$ & 0.45 & 0.62 & 0.001 \\
\cline { 2 - 5 } & $\tau_{L}(\mathrm{~s})$ & 95.9 & 85.1 & 10 \\
\hline
\end{tabular}

Table 3. Mechanical parameters for the BTINL model of AC (DiSilvestro, Zhu, Wong et al. 2001), in addition to the marked parameters $\left({ }^{*}\right)$ of Table 1.

\begin{tabular}{|c|c|c|c|}
\hline \multirow{4}{*}{ BTINL } & \multirow{3}{*}{$E_{e q}(\mathrm{MPa})$} & $E_{x}=E_{z}$ & 1.7 \\
\cline { 2 - 4 } & & $E_{y}$ & 0.421 \\
\cline { 2 - 4 } & \multirow{2}{*}{$v_{e q}$} & $v_{x y}=v_{y x}$ & 0.09 \\
\cline { 2 - 4 } & $v_{x z}=v_{y z}$ & 0.01 \\
\cline { 2 - 4 } & $G_{e q}(\mathrm{GPa})$ & $G_{x z}=G_{y z}$ & 0.84 \\
\cline { 2 - 4 } & \multicolumn{2}{|c|}{$k_{0}\left(\mathrm{~m}^{4} / \mathrm{N} \mathrm{s}\right)$} & $0.510^{-15}$ \\
\hline
\end{tabular}

Table 4. Main characteristics of the mesh of the patient specific TFJ model.

\begin{tabular}{|c|c|c|c|c|c|c|}
\hline \multirow[b]{2}{*}{ Part } & \multicolumn{5}{|c|}{ Elements } & \multirow{2}{*}{$\begin{array}{l}\text { Nodes } \\
\text { Number }\end{array}$} \\
\hline & Geometry & $\begin{array}{c}\text { Dimension } \\
(\mathrm{mm})\end{array}$ & Type & $\begin{array}{c}\text { Geometric } \\
\text { order }\end{array}$ & Number & \\
\hline Femur & \multirow{2}{*}{ Tetrahedral } & \multirow{2}{*}{$\begin{array}{l}1.5-4 \\
\text { (edge) }\end{array}$} & \multirow{2}{*}{ 3D stress } & \multirow{2}{*}{ Linear } & 5768 & 24636 \\
\hline Tibia & & & & & 5508 & 23590 \\
\hline LFAC & \multirow{4}{*}{ Hexahedral } & \multirow{4}{*}{$\begin{array}{c}1.5-2.5 \\
\text { (edge) } \\
+ \\
4 \text { layers }\end{array}$} & \multirow{4}{*}{$\begin{array}{l}\text { 3D } \\
\text { Stress/ } \\
\text { Pore } \\
\text { Fluid - } \\
\text { Stress }\end{array}$} & \multirow{4}{*}{ Quadratic } & 592 & 3143 \\
\hline MFAC & & & & & 924 & 4776 \\
\hline LTAC & & & & & 680 & 3547 \\
\hline MTAC & & & & & 684 & 3580 \\
\hline
\end{tabular}




\section{List of figures}

Figure 1. Simplified model of the TFJ: geometry of a quarter of a single compartment (a) and two examples of mesh (b).

Figure 2. Scheme of the BCs applied to simplified TFJ model. The global coordinate system is shown on the bottom-left.

Figure 3. Reconstruction of 3D geometry of a patient specific TFJ from CT (hard tissue) and MRI (soft tissue) scans (a) (with permission from M. Tuncer, Imperial College London); geometric parts of the model (b); model meshing (c).

Figure 4. Scheme of the BCs applied to patient-specific TFJ model. The global coordinate system is shown on the leftbottom.

Figure 5. Comparison of the AC models in terms of: vertical displacement of the RP (a); contact area (b); contact pressure (c); pore fluid pressure (d).

Figure 6. An example of the temporal evolution of the fluid/solid load support in the biphasic AC. The fluid/solid supports for two node paths at different depths in the AC thickness are compared.

Figure 7. Comparison of the global and the local parameters of the $M$ and the BPVNLb (simply labelled as "B") AC models, during 5 loading cycles: vertical displacement of the RP (a); contact area (b); contact pressure (c); pore fluid pressure (d).

Figure 8. Contour plots of the contact pressure of biphasic AC layers, at several instants during the walk. The contact pressure is higher for higher loads and decreases during time due to the load redistribution on wider contact areas.

Figure 9. Contour plots of Tresca stress of biphasic AC layers, at several instants during the walk. The von MisesTresca stress is higher for higher loads and decreases during time due to the stress relaxation.

Figure 10. Comparison of Tresca stresses predicted in the medial compartment implementing the BPVNLb (a) and M (b) models for the AC, at the first load peak of the first loading cycle. 